\title{
The Role of Highly Selective Implant Retention in the Infected Hip Arthroplasty
}

\author{
Moataz El-Husseiny MB BCh, MRCS, Dip Sport M, MD (Res), \\ Fares S. Haddad BSc, MCh(Orth), FRCS(Orth), MD (Res), FFSEM
}

Published online: 22 June 2016

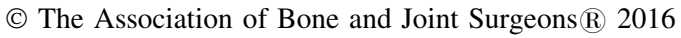

\begin{abstract}
Background There is debate around how to treat patients with periprosthetic joint infection of the hip. When there is an ingrown component on one side of the arthroplasty and a loose component on the other, treatment is typically revision of the entire construct. There is an argument to retain an ingrown implant in instances in which removal would result in severe bone damage. However, little has been reported on the likelihood of success with this approach.

Questions/purposes Among carefully selected patients presenting with an infected total hip arthroplasty (THA) who were treated with joint débridement and at least partial implant retention: (1) What proportion remained apparently free of infection at a minimum of 5 years of
\end{abstract}

Each author certifies that he or she, or a member of his or her immediate family, has no funding or commercial associations (eg, consultancies, stock ownership, equity interest, patent/licensing arrangements, etc) that might pose a conflict of interest in connection with the submitted article.

All ICMJE Conflict of Interest Forms for authors and Clinical Orthopaedics and Related Research ${ }^{\circledR}$ editors and board members are on file with the publication and can be viewed on request.

Clinical Orthopaedics and Related Research ${ }^{\mathbb{R}}$ neither advocates nor endorses the use of any treatment, drug, or device. Readers are encouraged to always seek additional information, including FDAapproval status, of any drug or device prior to clinical use.

Each author certifies that his or her institution approved the human protocol for this investigation, that all investigations were conducted in conformity with ethical principles of research, and that informed consent for participation in the study was obtained.

M. El-Husseiny ( $\square)$, F. S. Haddad

Department of Orthopaedics and Trauma, University College London Hospitals, 235 Euston Road, London NW1 2BU, UK

e-mail: moatazelhusseiny@gmail.com followup? (2) What were the Harris hip scores of patients thus treated?

Methods Between January 2000 and December 2010, a total of 293 patients were treated surgically at one hospital for a periprosthetic joint infection of the hip. Of these, $18(2.9 \%)$ were treated with an approach that retained either the femoral component or the acetabular component (the removed component was exchanged at this same single-stage procedure). During that time, the general indications for this approach were patients who had complex THAs with ingrown femoral stems or complex acetabular components that were well fixed with no evidence of loosening on radiographs and CT. Patients had to be free from chronic debilitating diseases, had not developed a tracking sinus, and had a positive microbial growth from the hip aspirate. In 12 of these patients, the ingrown cementless femoral component was kept in situ and the femoral head and acetabular component were exchanged. In six patients, complex acetabular reconstructions including augments and/or cages were left in situ, and femoral revision with liner exchange was performed. The technique included removal of the loose component, thorough débridement, synovectomy, and extensive lavage. The ingrown component, be it femoral or acetabular, was thoroughly cleaned, lavaged, and scrubbed. Once there was a clear field, redraping was carried out and new instruments were used to reimplant the other side. In all patients, intravenous antibiotics were used postoperatively for a minimum of 5 days and oral antibiotics for a minimum of 6 weeks based on serology, wound healing, and nutritional markers. None of the patients were lost to followup. Minimum followup was 5 years; median followup was 7.1 years (range, $5-9.9$ years).

Results Reinfection occurred in three patients at 3, 9, and 10 months; all were treated by two-stage revision. No reinfection was noted in the other cases. At latest followup, the mean Harris hip score was 78 (range, 46-89). 
Conclusions In some patients, staged revision of large and well-fixed components will result in bone damage and compromised function. These results suggest that partial implant retention and joint débridement may be an alternative for those patients who have complex well-fixed acetabular or femoral components, are not immunocompromised, have not developed sinus formation, and we were able to obtain a positive hip aspirate. We caution this technique should not be applied when patients have chronic illness such as diabetes or rheumatoid disease, have a negative hip aspirate for microorganisms, or show any signs of loosening on radiography, CT, or on intraoperative assessment. These results at a minimum of 5 years are reassuring in this small single-center series, but we suggest that the technique not be widely adopted until or unless larger groups of patients with longer term data have been studied.

Level of Evidence Level IV, therapeutic study.

\section{Introduction}

Periprosthetic infection occurs in $0.22 \%$ to $3 \%$ of patients who undergo arthroplasty [3, 4, 8, 9, 15, 21], and it is always devastating. Several techniques have been applied in an attempt to completely eradicate infection [14]. Two-stage revision arthroplasty remains the most commonly adopted method despite promising results from single-stage revision hip arthroplasty [2, 17, 20]. In both of these options, however, there is the potential for substantial bone loss and consequent morbidity from removal of well-fixed components. We present a new method in treating periprosthetic infection in highly selected patients, in which partial implant retention of well-fixed components was performed.

Because of the challenges associated with staged revision, a number of investigators have explored the possibility of retaining well-fixed components in the setting of two-stage treatment $[6,16,18]$. In this approach, the bearing surface is removed and replaced with a provisional bearing of antibiotic-laden PMMA, and all exposed portions of the affected implants are aggressively débrided. The most common scenario is removal of an acetabular component and retention of a long fully porous-coated or cemented femoral stem $[8,14,16,25]$. In our cohort of patients, who were not immunocompromised, with a known microorganism from hip aspiration and where implants were well fixed, careful assessment of the fixation of the components using radiographs, CT, and intraoperative assessment for stability by a senior fellowship-trained revision arthroplasty surgeon (FSH) and a careful decision to retain that component was taken with a view to allow less bone loss and improve morbidity. To our knowledge, there have been no studies using partial component retention as part of a definitive singlestage revision for periprosthetic joint infection in patients who fulfill strict selective criteria outlined here.

We therefore sought to determine among carefully selected patients presenting with an infected THA who were treated with joint débridement and at least partial implant retention: (1) What proportion remained apparently free of infection at a minimum of 5 years of followup? (2) What were the Harris hip scores of patients thus treated?

\section{Patients and Methods}

Patients included in the study had established periprosthetic joint infection and were treated in a specialized arthroplasty center by a single revision arthroplasty surgeon (FSH) who treats 50 infections/year. Two hundred ninety-three patients were treated for periprosthetic joint infection of the hip from January 2000 to December 2010 in our unit. Of these, 18 (2.9\%) patients were treated with an approach that retained either the femoral component or the acetabular component (the removed component was exchanged at this same single-stage procedure) (Tables 1 , 2). In 12 of the 18 patients, the ingrown cementless femoral component was kept in situ and the femoral head and acetabular component were exchanged. In six patients, complex acetabular reconstruction including augments and/or cages was left in situ, and femoral revision with liner exchange was performed.

Selection criteria included patients who had complex THAs with ingrown femoral stems or complex acetabular components that were well fixed with no evidence of loosening on radiographs and CT. Patients had to be free from chronic debilitating diseases such as renal failure and not be immunocompromised, nor diabetic, nor receive any longterm steroids. A positive microbial growth from the hip aspirate was required, enabling selection of microorganismsensitive antibiotics. If the patients fulfilled all these criteria, they were candidates to be included in this cohort study. The inclusion criteria depended on the nature of the fixation, history, and the difficulty that was perceived in removing an implant that was well fixed. No distinction was made among acute, chronic, or recurrent periprosthetic joint infections at the time of surgical decision-making. The decision was based primarily on the anatomic factors related to the implant and on the host and whether the host could withstand major surgery.

Once criteria for inclusion into the study were fulfilled, the decision to partially retain components was made according to strict pre- and intraoperative assessments.

Preoperative radiographs and serial radiographs were examined for any signs of loosening. CT scans were 


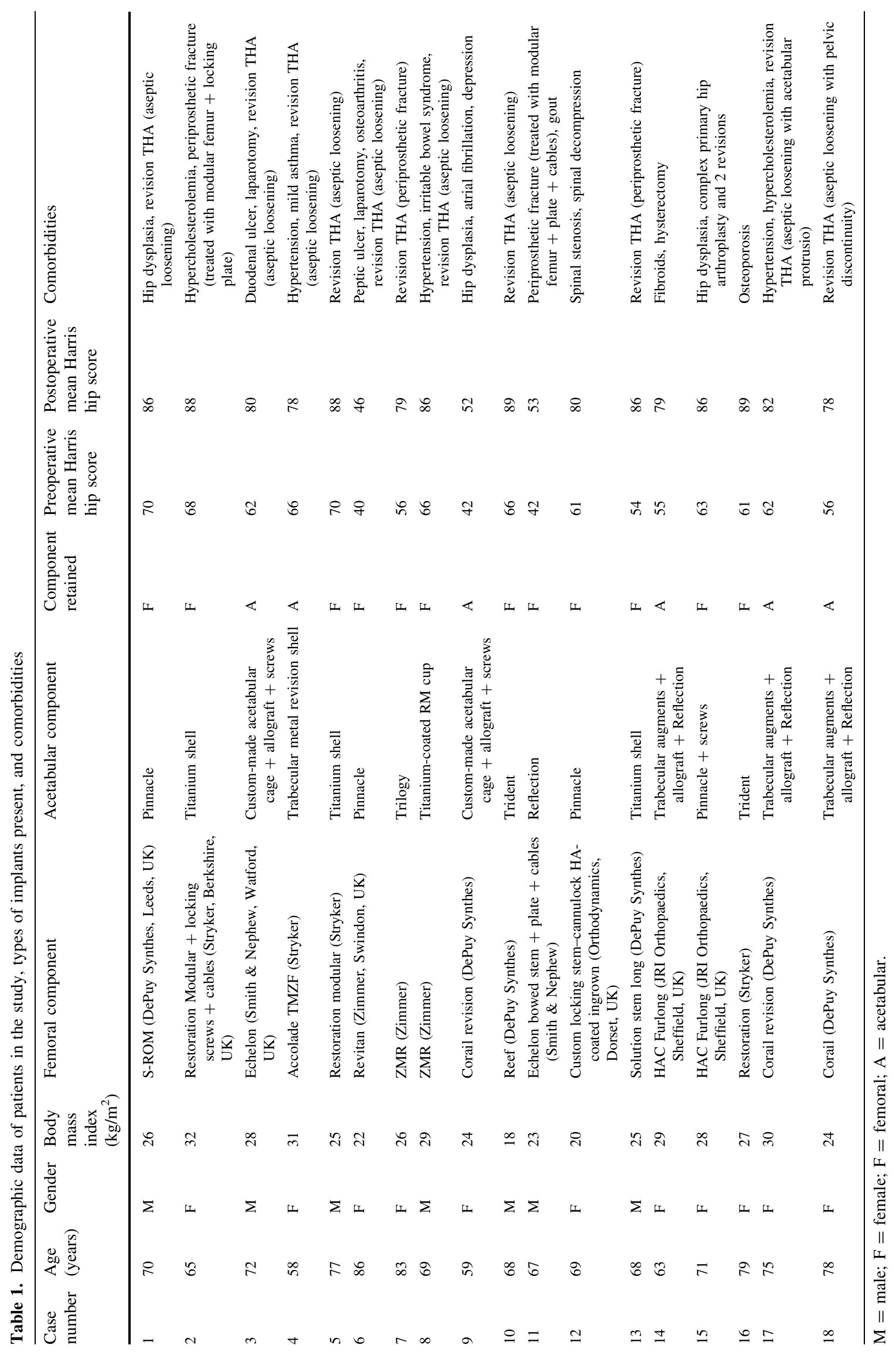


Table 2. Demographic data of patients in study and treatment times of surgical procedures performed

\begin{tabular}{ll}
\hline Demographic data & Value (range) \\
\hline Mean age at first-stage revision (years) (range) & $71(58-86)$ \\
Male:female (ratio) & $7: 11$ \\
Median time from primary procedure to partial single-stage revision (months) (range) & $56(32-145)$ \\
Median time from partial single-stage revision to latest followup (months) (range) & $61(24-97)$ \\
Mean Harris hip score (range) & $78(46-89)$ \\
\hline
\end{tabular}

carefully examined to identify any gaps in the bone-implant interface. In cases of acetabular components, complete fixation was required between the cup/cage and screws on one hand and the pelvic bone on the other. Similarly, in cases in which femoral component retention was planned, no gaps were identified in the bone-prosthesis implant. All scans were inspected in a multidisciplinary meeting in the presence of two revision arthroplasty, fellowship-trained surgeons (FSH, SO, RP), a musculoskeletal radiologist (MH-C), microbiologists (SM$\mathrm{J}, \mathrm{VG})$, a plastic surgeon (IY), and a physiotherapist (BB).

\section{Surgical Technique}

Intraoperative inspection of the implants and interfaces was carried out by a senior revision arthroplasty surgeon (FSH). Implant fixation was tested by attempting to move the implant in the AP and mediolateral directions and rotating it clockwise and counterclockwise. A component was removed if there were visible gaps at the bone-implant interface, wear damage on the implant, fretting corrosion around the trunnion, or movement of the prosthesis. If the implant was well fixed and showed no signs of loosening or damage, and the risks of excessive bone destruction were deemed to result in more morbidity than to retain implants, the implant was retained.

When partial revision hip arthroplasty was chosen, we performed thorough aggressive débridement and removal of any loose components. For acetabular components, the Explant Acetabular Cup Removal System (Zimmer Ltd, Swindon, UK) was used to extract the shell with specialized carbide drill bits or diamond-tipped burrs as needed to remove screws. In cases in which femoral components were removed, rongeurs were used to increase the gap between the stem and greater trochanter followed by flexible osteotomes (DePuy Synthes, Leeds, UK) to loosen the stem from endosteal bone. After component removal, we performed copious pulse lavage with $6 \mathrm{~L}$ normal saline and $1 \mathrm{~L}$ povidine-iodine. The wound was then packed with sterile swabs and the skin was closed with interrupted nylon. Occlusive dressings
Table 3. Infecting organisms cultured from specimens taken

\begin{tabular}{ll}
\hline Organism identified & Number of patients \\
\hline Methicillin-resistant Staphylococcus aureus & 3 \\
Methicillin-sensitive S aureus & 4 \\
Coagulase-negative Staphylococcus & 4 \\
Pseudomonas & 3 \\
Enterobacter & 2 \\
Streptococcus & 2 \\
\hline
\end{tabular}

were applied and drapes, surgical gowns, and instruments were discarded.

Redraping and new instruments were used in the second part of the procedure. In cases of contained bone loss on the acetabular side, $4 \mathrm{~g}$ vancomycin powder was spread evenly in the acetabulum and a large cementless porouscoated, press-fit acetabular cup was inserted. If this was not possible, antibiotic-loaded bone graft, cages, and augments were also used. The retained femoral trunnion was covered with an adaptor sleeve and a ceramic head was used with a polyethylene liner.

Postoperatively, all patients had intravenous antibiotics administered for at least 5 days during which inflammatory and nutritional markers were closely monitored. Drains were left for 48 hours before removal. The wound was inspected on the fifth day, and if the wound had settled completely, with a downward trend of inflammatory markers, patients were switched to oral antibiotics to which the organism was sensitive for 6 weeks. In cases in which a downward trend of inflammatory markers was not seen, close observation of the wound with continuation of intravenous antibiotics according to culture sensitivities was done in addition to supplementary antibiotics sensitive to grown microorganisms, as advised by the microbiologists until inflammatory markers declined.

Hip aspiration was positive in all patients as the inclusion criteria required (Table 3); three patients had methicillin-resistant Staphylococcus aureus, four had methicillin-sensitive $S$ aureus, four had coagulase-negative Staphylococcus, three Pseudomonas, two Streptococcal 
species, and two Enterobacter. None of the patients were lost to followup. Our recurrence rate of infection was $17 \%$ (three of 18). There were four cases of mortality at 5 years, three of which were infection-free and none of which were related to periprosthetic infection.

\section{Followup}

None of the patients were lost to followup. Minimum followup was 5 years; median followup was 7.1 years (range, 5-9.9 years).

Patients were seen in the outpatient clinic at 6 weeks, 3 months, 6 months, 9 months, 12 months, 18 months, 24 months, and annually thereafter as part of a standard protocol. Patients underwent blood investigations before their appointment and results were available at the consultation. Harris hip scores were assessed at followup. All patients attended their appointments. A research nurse called all patients before their appointments and if they were not able to attend that date, another one was offered. Recurrent infection was defined as failure of inflammatory markers to settle the patients to the patient's baseline or radiographic changes suggestive of infection.

\section{Results}

Control of overt periprosthetic joint infection was equivalent with a single-stage partial revision to that of two-stage partial revision arthroplasty [6]. Of our 18 patients, three patients developed recurrent infection. These occurred at 3 , 6 , and 9 months after the débridement. All three received two-stage revision THA. One of these patients died 31 months after the procedure; there were three other deaths in this series (at 51, 62, and 85 months after surgery), all among patients who were apparently without recurrent infection. None of the deaths were associated with the surgical intervention.

The mean Harris hip score was fair at 78 (range, 46-89) at latest followup with a mean Harris hip score pain component of 36 (range, 10-44) with a higher score representing better pain results.

\section{Discussion}

Periprosthetic joint infection remains one of the most challenging complications facing hip arthroplasty, leaving a devastating effect on patients with variable morbidity. Two-stage revision hip arthroplasty involves considerable morbidity and time out of work. Meanwhile, single-stage revision seems an attractive option to reduce cost, morbidity, and prolonged rehabilitation requirements. While centers have tried to retain implants in periprosthetic joint infection, we were keen to explore whether singlestage partial retention of well-fixed implants would yield reasonable outcomes in selected cases. We had 15 patients who had no recurrence of infection out of 18 treated with partial retention of implants and we continue to monitor them closely.

Our study has several limitations. This study did not have a comparison or control group alongside the study group. The cases were selected if they met all inclusion criteria; not immunocompromised, no sinus track, known organism, complex implant that was well fixed with radiography, CT, and with intraoperative assessment by an experienced revision arthroplasty surgeon. Decision-making involved a multidisciplinary team to establish how to best manage each patient. Thus, the inclusion criteria for the described approach were highly selective and subjective. We also note the possibility of assessment bias. Although 15 patients have had no recurrence, they may well do so in the future and are currently under close surveillance. Because the study was carried out before creation of Musculoskeletal Infection Society criteria, and repeat aspirations were not done, some of our patients may harbor covert infection. The small number of cases in this study remains a major limitation. Even with a high volume of periprosthetic joint infection referrals to our center, we could only include 18 patients in this cohort. We were unable to stratify the data to the virulence of the infecting organism, duration of infection, and subdivision of acetabular and femoral components.

Our results cannot be directly compared with other total single-stage $[5,7,20,24]$ and two-stage revision hip arthroplasty studies for infection [1, 11, 12, 19, 22]. We have a highly selected cohort of patients who fulfill different inclusion criteria, and our study size is too small to reach definitive conclusions. A multicenter randomized controlled trial such as the INFORM trial would be of substantial benefit [23]. A recent meta-analysis from that group showed reinfection rates of $8.2 \%$ and $7.9 \%$ in singlestage and two-stage hip arthroplasty, respectively. They concluded that reinfection rates remained similar when grouped by several study and population-level characteristics [10]. A similar study is required for partial singlestage and two-stage hip arthroplasty. There is a limited scope of bacteriology; surgeon's technique and experience play a role in determining likely cases that can be included. Longer term followup is needed to evaluate the success of this treatment modality.

Several two-stage partial hip arthroplasty case series have been reported recently in the medical literature with recurrence of infection between two of 19 patients and four of 19 patients [6, 13, 16, 18]. Partial one-stage revision 
arthroplasty retains bone stock, hence preventing fixation compromise. Extensive soft tissue dissection devascularizes the proximal femur, predisposing to osteomyelitis and further infection. On the other hand, any residual infected tissues left in any débridement procedure represent a catastrophe that can only lead to failure. Use of specialized acetabular removal devices allows minimal bone loss with simple acetabular components. However, complex acetabular components and femoral explant remain a challenge and can be complicated as a result of improved biologic fixation of implants and cementing techniques.

Our study shows recurrence of infection in three of 18 at a minimum of 5 years followup for a highly selected group of patients who fulfilled a strict inclusion criterion. Ekpo et al. [6] retained one component in a two-stage revision arthroplasty procedure, showing recurrence of infection in two of 19 at a minimum of 2 years followup (range, 2-11 years). Morley et al. [18] retained the original well-fixed femoral cement mantle in 15 patients with infected hip arthroplasty. Two patients had positive microbiology results at the second stage requiring 6 weeks of antibiotics. One of these two developed recurrent infection and needed further revision surgery. Their mean followup was 6.8 years. In addition, Lee et al. [11] retained well-fixed cementless femoral stems in 17 patients who underwent two-stage revision hip arthroplasty for infection. Two developed recurrent infection and needed revision procedures. Their mean followup was 4 years (range, 2-8 years).

We assessed the functional outcome in our patients. The mean Harris hip score in our study was fair at 78 (range, 46-89) at latest followup with a mean Harris hip score pain component of 36 (range, 10-44) with a higher score representing better pain results. Ekpo et al. [6] used the mean Harris hip score to assess their patients' function and was 68 (range, 31-100; best score is 100).

Our study shows that partial single-stage partial hip arthroplasty with aggressive débridement and retention of well-fixed femoral or acetabular components can be successful in treatment of highly selected periprosthetic joint infections in patients with well-fixed complex acetabular or complex modular femoral stems, who are not immunocompromised or who have chronic illnesses and who have a positive organism on hip aspiration. Devascularization of adjacent tissues, challenging prolonged fixation revision procedures, may be avoided with less extensive revision surgery, preservation of bone, and less morbidity to the patient. We believe longer followup and multicenter studies are required to validate our results before wider use.

Acknowledgments We thank Dr Stephen Morris-Jones and Dr Vanya Gant, microbiology consultants at University College London Hospitals, for providing advice regarding use and monitoring of antibiotics for our patients and attending our multidisciplinary meetings. We also thank Dr Margaret Hall-Craggs, musculoskeletal radiologist at University College London Hospitals, for reviewing the imaging and providing support for our department. We thank Dr Ibby Younis, plastic consultant, and Ms Bindyia Bhandari, physiotherapist, for their constructive contributions in the joint infection multidisciplinary meetings. We also thank Dr Sam Oussedik and Dr Rahul Patel, orthopaedic consultants at University College London Hospitals, for their contribution in the joint infection multidisciplinary meetings where these patients were discussed.

\section{References}

1. Berend KR, Lombardi AV, Jr., Morris MJ, Bergeson AG, Adams JB, Sneller MA. Two-stage treatment of hip periprosthetic joint infection is associated with a high rate of infection control but high mortality. Clin Orthop Relat Res. 2013;471:510-518.

2. Beswick AD, Elvers KT, Smith AJ, Gooberman-Hill R, Lovering A, Blom AW. What is the evidence base to guide surgical treatment of infected hip prostheses? Systematic review of longitudinal studies in unselected patients. BMC Med. 2012;10:18.

3. Blomfeldt R, Kasina P, Ottosson C, Enocson A, Lapidus LJ. Prosthetic joint infection following hip fracture and degenerative hip disorder: a cohort study of three thousand, eight hundred and seven consecutive hip arthroplasties with a minimum follow-up of five years. Int Orthop. 2015;39:2091-2096.

4. Bozic KJ, Ries MD. The impact of infection after total hip arthroplasty on hospital and surgeon resource utilization. $J$ Bone Joint Surg Am. 2005;87:1746-1751.

5. Darley ES, Bannister GC, Blom AW, Macgowan AP, Jacobson SK, Alfouzan W. Role of early intravenous to oral antibiotic switch therapy in the management of prosthetic hip infection treated with one- or two-stage replacement. J Antimicrob Chemother. 2011;66:2405-2408.

6. Ekpo TE, Berend KR, Morris MJ, Adams JB, Lombardi AV Jr. Partial two-stage exchange for infected total hip arthroplasty: a preliminary report. Clin Orthop Relat Res. 2014;472:437-448.

7. Engesaeter LB, Dale H, Schrama JC, Hallan G, Lie SA. Surgical procedures in the treatment of 784 infected THAs reported to the Norwegian Arthroplasty Register. Acta Orthop. 2011;82:530537.

8. Gundtoft PH, Overgaard S, Schonheyder HC, Moller JK, Kjaersgaard-Andersen $\mathrm{P}$, Pedersen AB. The 'true' incidence of surgically treated deep prosthetic joint infection after 32,896 primary total hip arthroplasties: a prospective cohort study. Acta Orthop. 2015;86:326-334.

9. Huotari K, Peltola M, Jamsen E. The incidence of late prosthetic joint infections: a registry-based study of 112,708 primary hip and knee replacements. Acta Orthop. 2015;86:321-325.

10. Kunutsor SK, Whitehouse MR, Blom AW, Beswick AD. Reinfection outcomes following one- and two-stage surgical revision of infected hip prosthesis: a systematic review and metaanalysis. PLoS One.2015;10:e139166.

11. Lee YK, Lee KH, Nho JH, Ha YC, Koo KH. Retaining well-fixed cementless stem in the treatment of infected hip arthroplasty. Acta Orthop. 2013;84:260-264.

12. Leung F, Richards CJ, Garbuz DS, Masri BA, Duncan CP. Twostage total hip arthroplasty: how often does it control methicillinresistant infection? Clin Orthop Relat Res. 2010;469:1009-1015.

13. Li P, Hou M, Zhu ZQ, Shi ZJ. Cementless revision for infected hip arthroplasty: an 8.6 years follow-up. Orthop Surg. 2015;7:3742.

14. Lieberman JR, Callaway GH, Salvati EA, Pellicci PM, Brause BD. Treatment of the infected total hip arthroplasty with a twostage reimplantation protocol. Clin Orthop Relat Res. 1994;301:205-212. 
15. Lindgren JV, Gordon M, Wretenberg P, Karrholm J, Garellick G. Validation of reoperations due to infection in the Swedish Hip Arthroplasty Register. BMC Musculoskelet Disord. 2014;15:384.

16. Lombardi AV Jr, Berend KR, Adams JB. Partial two-stage exchange of the infected total hip replacement using disposable spacer moulds. Bone Joint J. 2014;96:66-69.

17. Masri BA, Panagiotopoulos KP, Greidanus NV, Garbuz DS, Duncan CP. Cementless two-stage exchange arthroplasty for infection after total hip arthroplasty. J Arthroplasty. 2007;22:7278

18. Morley JR, Blake SM, Hubble MJ, Timperley AJ, Gie GA, Howell JR. Preservation of the original femoral cement mantle during the management of infected cemented total hip replacement by two-stage revision. J Bone Joint Surg Br. 2012;94:322327.

19. Neumann DR, Hofstaedter T, List C, Dorn U. Two-stage cementless revision of late total hip arthroplasty infection using a premanufactured spacer. J Arthroplasty. 2011;27:1397-1401.

20. Oussedik SI, Dodd MB, Haddad FS. Outcomes of revision total hip replacement for infection after grading according to a standard protocol. J Bone Joint Surg Br. 2010;92:1222-1226.
21. Pulido L, Ghanem E, Joshi A, Purtill JJ, Parvizi J. Periprosthetic joint infection: the incidence, timing, and predisposing factors. Clin Orthop Relat Res. 2008;466:1710-1715.

22. Romano CL, Romano D, Albisetti A, Meani E. Preformed antibiotic-loaded cement spacers for two-stage revision of infected total hip arthroplasty. Long-term results. Hip Int. 2012;22(Suppl 8):S46-53.

23. Strange $S$, Whitehouse MR, Beswick AD, Board T, Burston A, Burston B, Carroll FE, Dieppe P, Garfield K, Gooberman-Hill R, Jones S, Kunutsor S, Lane A, Lenguerrand E, MacGowan A, Moore A, Noble S, Simon J, Stockley I, Taylor AH, Toms A, Webb J, Whittaker JP, Wilson M, Wylde V, Blom AW. Onestage or two-stage revision surgery for prosthetic hip joint infection - the INFORM trial: a study protocol for a randomised controlled trial. Trials.2016;17:90.

24. Yoo JJ, Kwon YS, Koo KH, Yoon KS, Kim YM, Kim HJ. Onestage cementless revision arthroplasty for infected hip replacements. Int Orthop. 2009;33:1195-1201.

25. Zurcher-Pfund L, Uckay I, Legout L, Gamulin A, Vaudaux P, Peter R. Pathogen-driven decision for implant retention in the management of infected total knee prostheses. Int Orthop.2013;37:1471-1475. 